\title{
Temporal Changes in Swallowing Function Caused by a Palate Covering
}

\author{
Junichi Furuya, DDS, PhD, Atsushi Suzuki, DDS, PhD, Tetsuya Suzuki, DDS, PhD, \\ Nobusuke Oda, DDS, PhD, and Takuya Kobayashi, DDS, PhD
}

Department of Removable Prosthodontics, School of Dentistry, Iwate Medical University, Morioka, Japan

\section{Clinical significance}

Use of prostheses such as complete dentures is often avoided in dysphagia rehabilitation for the elderly due to the effects of the palate covering. However, the influence of the palate covering is relieved over a short time (approximately 7 days), suggesting that the commonly used approach should be reconsidered.

\begin{abstract}
Purpose: The purpose of this study was to elucidate the temporal relationship between habituation to a palate covered by a prosthesis and swallowing function.

Materials and methods: Ten healthy adults were selected as subjects. Tongue pressure on an experimental palatal plate with four pressure sensors was recorded simultaneously with surface electromyography measurements of suprahyoid muscles during swallowing of $3 \mathrm{ml}$ of water. Measurements were performed 6 times: immediately after placement of the experimental palatal plate (Day 0), and on Day 1, Day 3, Day 7, Day 10, and Day 14. Temporal changes were analyzed in terms of the order of EMG activity and tongue pressure, and the duration for total swallowing (DTS), oral preparatory stage (DOPS), oral stage (DOS), and pharyngeal stage (DPS).

Results: Onset of activity of the suprahyoid muscles was observed significantly earlier in all regions compared to onset of tongue pressure. Onset of tongue pressure in the posterior region was significantly delayed compared to onset of tongue pressure in another region. These results remained constant over the measurement period. DTS and DOS were significantly shortened on and after Days 3 and 7, respectively, compared to the respective values on Day 0. DOPS was significantly shortened on Day 7, compared to Day 0 and 1. DPS showed no significant change over time.
\end{abstract}

Corresponding to: Dr Junichi Furuya

Department of Removable Prosthodontics, School of Dentistry, Iwate Medical University

19-1 Uchimaru, Morioka 020-8505, Japan

Tel: +81-19-651-5111, Fax: +81-19-652-3820

E-mail: junfuru@iwate-med.ac.jp

Received on November 15, 2007/ Accepted on April 14, 2008
Conclusion: The time required for swallowing shows a tendency to decrease over time as a subject becomes accustomed to a palate covering. This was mainly due to shortening of the oral stage.

Key words: swallowing, tongue pressure, palate covering, denture, habituation

\section{Introduction}

The elderly often require dentures that cover the palate due to their few remaining teeth, ${ }^{1}$ and often have reduced swallowing function due to age or underlying diseases such as cerebrovascular diseases and asymptomatic cerebral infarction..$^{2-6}$ However, dentures are often avoided in the elderly especially with dysphagia due to their ill-fitting dentures and disadvantages such as the uncomfortable sensation caused by the palate covering. Therefore, to elucidate the relationship between the palate $\mathrm{cov}^{-}$ ering and swallowing function is an most important issue. In the oral preparatory stage and oral stage of swallowing, ${ }^{7}$ the tongue is responsible for putting food in the mouth, mastication, bolus formation, and transport to the pharynx. Kahrilas et $\mathrm{al}^{8}$ reported that the most important factors in the oral stage are that the tongue apex transports the bolus to the pharynx by appropriate fixation, acts as an anchor of the wavelike movement of the tongue, makes a concave shape in the midline of the dorsum of the tongue while the lateral region of the tongue contacts the lateral region of the palate, and subsequently makes the dorsum of the tongue contact the anterior to posterior region of the palate. The importance of appropriate contact between the tongue and the palate was pronounced, and tongue dynamics have been studied using ultrasonography ${ }^{9}$ and contact pressure sensors $^{10}$ with advanced ME technology.

A palate-covering prosthesis may have a structural and physical influence on swallowing func- 
tion due to a reduction in the oral cavity volume or sensory deprivation, and may particularly affect tongue function ${ }^{6,11,12}$ in the oral preparatory stage and oral stage of swallowing. However, the detailed effects on swallowing function caused by covering a palate with dentures are unknown. In general, patients may become accustomed to the palate being covered by a prosthesis in one week, in terms of articulation and foreign-body sensation. ${ }^{13-18}$ The relationship between habituation to a palate covering and swallowing function have not been examined except by Ando et al ${ }^{19}$ who reported the relationship between swallowing following mastication and habituation to an experimental palatal plate. Furthermore, which stages were influenced by palate covering in 5 stages (anticipatory stage, oral preparatory stage, oral stage, pharyngeal stage, esophageal stage) of swallowing process are unknown. Therefore, we measured surface electromyography (EMG) of the suprahyoid muscles and tongue pressure against the palate in subjects with healthy dentition who wore experimental palatal plates for 2 weeks, in order to clarify the temporal changes in swallowing function with habituation to a covered palate.

\section{Materials and methods}

\section{Subjects}

The subjects were 5 males and 5 females with a mean age of $28.1 \pm 3.0$ years old. All subjects had healthy dentition and no problems with their swallowing function. Informed consent was obtained from all subjects according to the protocol approved by the Ethics Committee of Iwate Medical University, School of Dentistry (Approval No.01055).

\section{Experimental palatal plate}

Impressions of the upper jaws of the subjects were taken using silicon impression material (Exahiflex, GC, Tokyo, Japan) to make plaster models. Two experimental palatal plates were made using heat-curing denture base resin (Acron, GC) using the standard manufacturing procedure for dentures. One was used to measure tongue pressure against the palate during swallowing, and the other was used for the subjects to get accustomed to the palate covering. Each experimental palatal plate had a $2-\mathrm{mm}$ thickness and uniformly $\mathrm{cov}^{-}$ ered the whole hard palate (Fig. 1). The anterior and lateral margins contacted the lingual cervical regions of the teeth and the posterior margin was

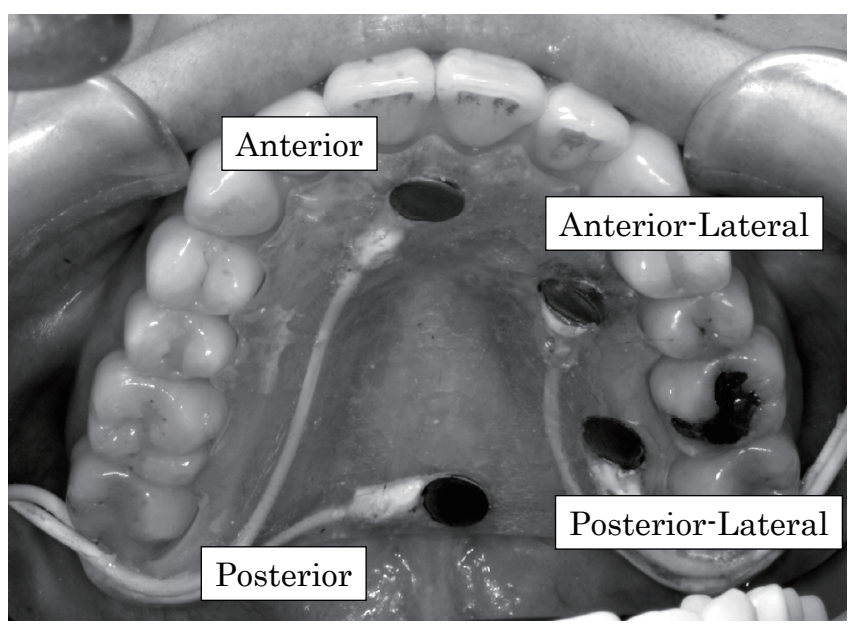

Fig. 1 Experimental Palatal Plate for measuring tongue pressure and position of pressure sensors. Anterior: $3 \mathrm{~mm}$ behind the incisal papilla, AnteriorLateral: $3 \mathrm{~mm}$ lingual the contact between the first and second premolar, Posterior-Lateral: $3 \mathrm{~mm}$ lingual the contact between first and second molars, Posterior: the midline of the hard palate $3 \mathrm{~mm}$ anterior to the posterior margin.

determined based on the Ah-line. Compact pressure sensors with a diameter of $6 \mathrm{~mm}$ and a thickness of $0.6 \mathrm{~mm}$ (PS-1KC-S-S1, Kyowa Electrical Instruments, Tokyo, Japan) were placed into the experimental palatal plate using a self-curing resin (UNIFAST II, GC) to measure tongue pressure during swallowing in four palatal regions: the anterior, anterior-lateral, posterior-lateral, and posterior parts of the palate. For each sensor, a lead with a diameter of $1 \mathrm{~mm}$ was placed into the experimental palatal plate, and ran from distal of the second molar through the oral vestibule and came out from the angle of mouth.

\section{Measurement of swallowing function}

The subjects sat upright at a 90-degree angle and the Frankfort plane was adjusted parallel to the floor using a headrest. Swallowing function was evaluated by surface EMG of the suprahyoid muscles and tongue pressure against the palate. A polygraph (Polygraph 361, Japan GE Marquette, Tokyo, Japan) and bipolar surface $\mathrm{Ag}-\mathrm{AgCl}$ electrodes with a diameter $8 \mathrm{~mm}$ were used to record surface EMG activity of the suprahyoid muscles from the anterior belly of the right digastric muscle at an interelectrode distance of $20 \mathrm{~mm}$. The experimental palatal plate was placed in the upper jaw of each subject and output from the compact pressure sensor was amplified using a dynamic strain recorder (DPM-611A, Kyowa Electronic In- 
struments) to measure tongue pressure.

The subjects swallowed $3 \mathrm{ml}$ of water at a temperature of $37^{\circ} \mathrm{C}$. They were asked to put the water on the anterior floor of the oral cavity proper and then close their mouth to hold and swallow the water according to instructions. The same process was repeated three times, and the overall measurement procedure was performed 6 times: immediately after placement of the experimental palatal plate (Day 0), and on Day 1, Day 3, Day 7, Day 10, and Day 14. After the first measurement, the subjects wore another experimental palatal plate each day except for 8 hours at night to get accustomed to the palate covering. Data for EMG and tongue pressure were converted from analog to digital using an A/D converter (PowerLab16/30, AD Instruments Japan, Tokyo, Japan) with a frequency of $2 \mathrm{kHz}$, which was synchronized and recorded on a PC. The data (Fig. 2) were analyzed for each measurement day with software (Chart ver.5.0, AD Instruments Japan).

Onset of tongue pressure on anterior, anteriorlateral, posterior-lateral and posterior regions of the palate $\left(\mathrm{TP}_{\mathrm{A}}\right.$ on, $\mathrm{TP}_{\mathrm{AL}}$ on, $\left.\mathrm{TP}_{\mathrm{PL}} \mathrm{On}, \mathrm{TP}_{\mathrm{P}} \mathrm{On}\right)$ was determined from the onset of activity of the suprahyoid muscles (SHon), and the order of onset of tongue pressure was investigated on each measurement day. The duration of EMG activity of the suprahyoid muscles was defined as the time for total swallowing (DTS) and temporal changes were investigated. To investigate which period in total swallowing was effected by habituation to the palate covering, DTS was divided into 3 stages, the oral preparatory stage, oral stage, and pharyngeal stage, and temporal changes in these time periods were also assessed. The duration of the oral preparatory stage (DOPS) was defined as the time from onset of SHon to tongue contact with the anterior region of the palate $\left(\mathrm{TP}_{\mathrm{A}} \mathrm{On}\right)$, the duration of the oral stage (DOS) was defined as the time from $\mathrm{TP}_{\mathrm{A}}$ on to the point of maximum tongue pressure in the posterior region ( $\left.\mathrm{TP}_{\mathrm{P}} \mathrm{max}\right)$, and the duration of the pharyngeal stage (DPS) was defined as the time from $\mathrm{TP}_{\mathrm{P}}$ max to the termination of activity of the suprahyoid muscles (SHoff).

\section{Statistical analysis}

Statistical analysis was performed using SPSS ver.15.0J (SPSS Japan Inc, Tokyo, Japan). To elucidate the temporal changes in the order of EMG activity of the suprahyoid muscles and tongue pressure, and temporal changes in the duration for swallowing, statistical analysis was performed

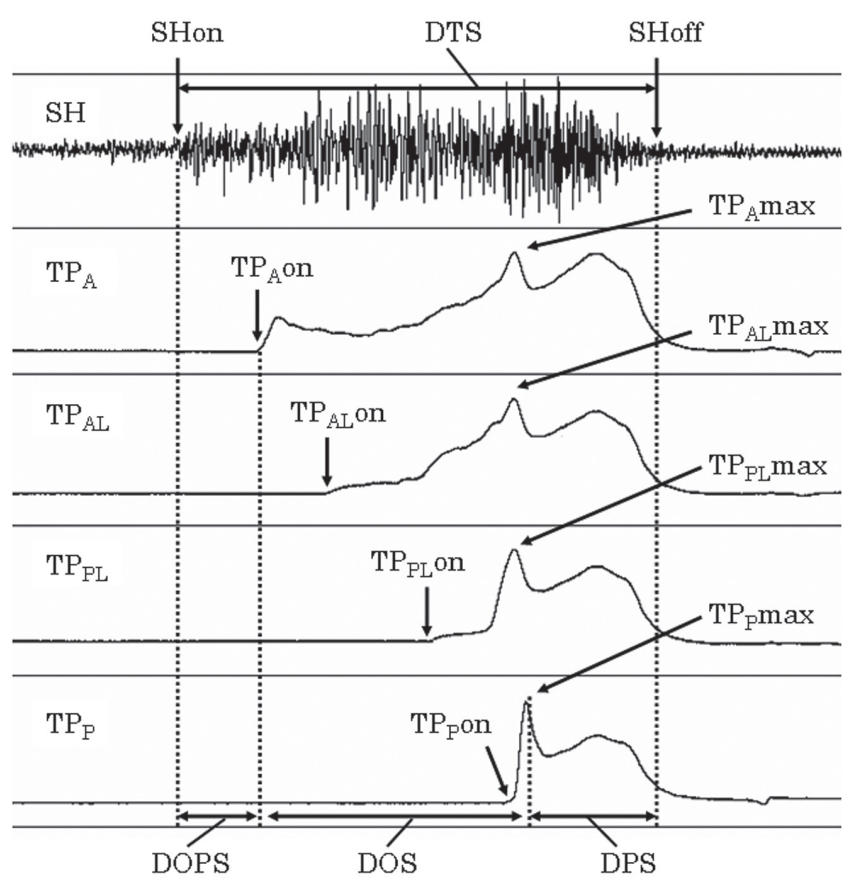

Fig. 2 Time points and duration of measured data. $\mathrm{SH}$ : EMG activity of suprahyoid muscles, TPA, TPAL, TPPL, TPP: Tongue pressure on anterior, anterior-lateral, posterior-lateral and posterior regions of the palate. SHon: Onset of EMG activity of suprahyoid musucles, SHoff: Termination of EMG activity of suprahyoid musucles. TPAon, TPALon, TPplon, TPpon: Onset of tongue pressure on anterior, anterior-lateral, posterior-lateral and posterior regions of the palate. TPAmax, TPALmax, TPplmax, TPpmax: Time points of maximum tongue pressure on anterior, anterior-lateral, posterior-lateral and posterior regions of the palate.

DTS: duration of total swallowing, DOPS: duration of oral preparatory stage, DOS: duration of oral stage, DPS: duration of pharyngeal stage.

using one-way repeated measures ANOVA, and a multiple comparison was conducted by Tukey's test, with a significance level of 0.05 .

\section{Results}

\section{Order of onset of EMG activity of the suprahy- oid muscles and tongue pressure}

On all measurement days, SHon occurred significantly earlier in all regions compared to onset of tongue pressure. (Table 1, Fig.3) Tongue contact started in the anterior or anterior-lateral region of the palate, followed by the posterior-lateral region. Tongue contact with the posterior region was significantly delayed. These results remained constant on all measurement days. 
Table 1 Order of onset of suprahyoid muscles and tongue pressure.

\begin{tabular}{rccccc}
\hline Day & SHon & TPAon & TPALon & TPPLon & TPPon \\
\hline 0 & $0.00 \pm 0.00^{\mathrm{a}}$ & $0.31 \pm 0.16$ & $0.33 \pm 0.14$ & $0.39 \pm 0.22$ & $0.68 \pm 0.16^{\mathrm{b}}$ \\
1 & $0.00 \pm 0.00^{\mathrm{a}}$ & $0.32 \pm 0.16$ & $0.32 \pm 0.07$ & $0.39 \pm 0.15$ & $0.64 \pm 0.14^{\mathrm{b}}$ \\
3 & $0.00 \pm 0.00^{\mathrm{a}}$ & $0.29 \pm 0.17$ & $0.27 \pm 0.10$ & $0.32 \pm 0.14$ & $0.63 \pm 0.16^{\mathrm{b}}$ \\
7 & $0.00 \pm 0.00^{\mathrm{a}}$ & $0.20 \pm 0.10$ & $0.22 \pm 0.10$ & $0.26 \pm 0.13$ & $0.50 \pm 0.10^{\mathrm{b}}$ \\
10 & $0.00 \pm 0.00^{\mathrm{a}}$ & $0.22 \pm 0.12$ & $0.22 \pm 0.07$ & $0.26 \pm 0.10$ & $0.52 \pm 0.10^{\mathrm{b}}$ \\
14 & $0.00 \pm 0.00^{\mathrm{a}}$ & $0.22 \pm 0.11$ & $0.18 \pm 0.06$ & $0.23 \pm 0.10$ & $0.48 \pm 0.10^{\mathrm{b}}$ \\
\hline
\end{tabular}

Unit: sec, Mean \pm SD

SHon was set to 0 sec.

${ }^{a}$ SHon was earlier than TPAon, TPALon, TPPLon, TPPon. $(p<0.05)$

${ }^{b} \mathrm{TP}$ Pon was later than TPAon, TPALon, TPPLon. $(p<0.05)$

SHon: Onset of EMG activity of suprahyoid musucles. TPAon,

TPALon, TPPLon, TPPon: Onset of tongue pressure on anterior,

anterior-lateral, posterior-lateral, posterior region of the palate.

\section{Temporal changes in the duration of swallow- ing}

Figures 4-7 show the temporal changes in DST, DOPS, DOS, and DPS. A tendency for shortening of DTS and DOS was noted over time. DTS on and after Day 3 was significantly shortened compared to Day 0, and no significance shortening was noted among DTS on and after Day 7. DOS on and after Day 7 was significantly shortened, compared to Day 0. DOPS on Day 7 was significantly shortened, compared to Day 0 and 1. DPS showed no significant change over time.

\section{Discussion}

\section{Definition of swallowing stages}

SHon was defined as the onset of swallowing movement, because tongue apex movement, activity of the tongue base, hyoid bone elevation, and activity of suprahyoid muscles start almost simultaneously at the onset of swallowing. ${ }^{20,21}$ The swallowing movement process has been suggested to differ from the command swallow of a liquid or the chew-swallow in the feeding sequence for food. ${ }^{22}$ In this study, the command swallow of water was used, and the time required for swallowing was classified into three stages based on typical swallowing models, such as "the five-stage process of ingestion". 7 The definition of the time point that defines the end of the oral stage and the start of the pharyngeal stage varies among studies. This is often considered to be the time point at which the bolus head passes the posterior arch of the palate in studies using Videofluorography(VF). ${ }^{2,20} \mathrm{How}^{-}$ ever, Shaw et $\mathrm{al}^{3}$ suggested that this point might be depended on the subject's ability to control the

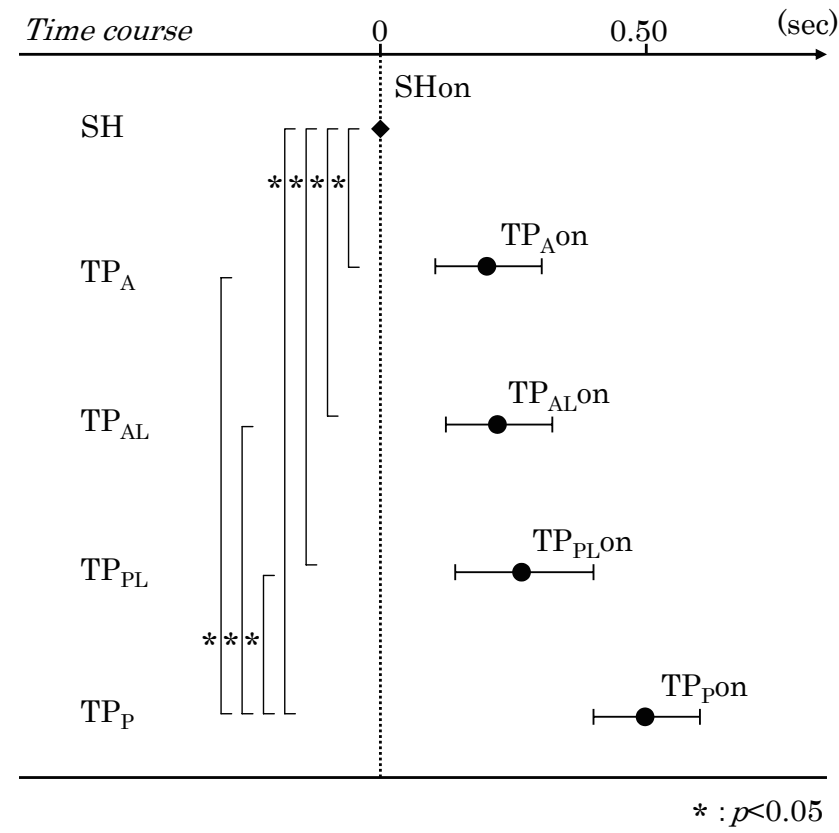

Fig. 3 A schema of the typical pattern of the order of EMG activity onset of the suprahyoid muscles and tongue pressure (Data on Day 7).

bolus within the mouth, thereby recommended the time point which the bolus tail passes the posterior arch of the palate as the definition. Regarding tongue pressure, Taniguchi et $\mathrm{al}^{23}$ found that $\mathrm{VF}$ and tongue pressure were synchronized, with the start of tongue contact in the posterior region matching the time the bolus head passes the pendulum of the palate, and the maximum tongue pressure in the posterior region coinciding with the time the bolus tail passes the pendulum of the palate. In this study, all subjects showed a similar pattern in which the pressure rapidly reached a peak immediately after the start of tongue contact in the posterior region. Therefore, the point of maximum tongue pressure in the posterior region of the palate was defined as the end of the oral stage and the start of the pharyngeal stage in this study.

\section{Order of onset of EMG activity of the suprahy- oid muscles and tongue pressure}

Similarly to a previous study ${ }^{24}$ using EMG, the activity of the suprahyoid muscles started at the earliest stage in this study. Subsequently, tongue contact was observed in the anterior region of the palate, followed by the anterior-lateral region and the posterior-lateral region. Tongue contact in the posterior region was the last to begin. In addition, tongue contact in the anterior-lateral region start- 


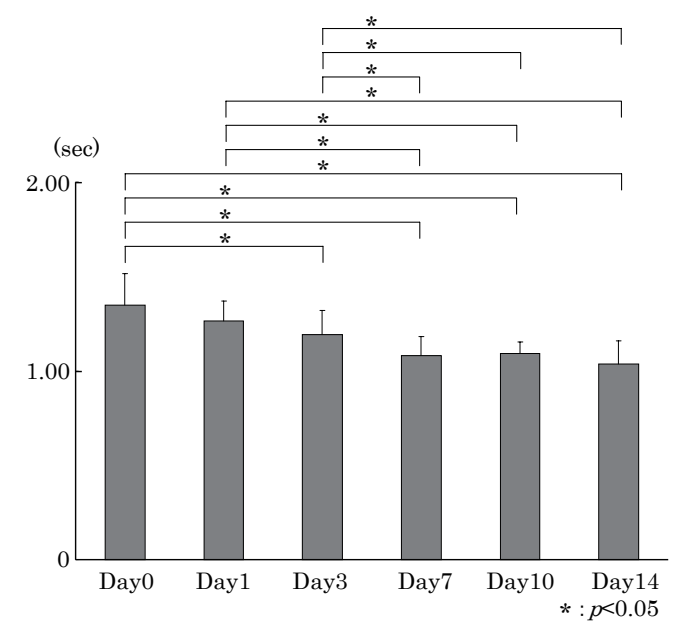

Fig. 4 Temporal change in duration of total swallowing (DTS).

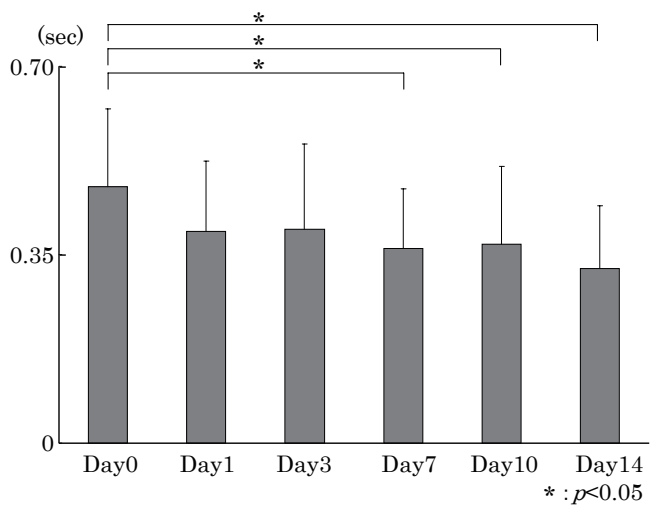

Fig. 6 Temporal change in duration of oral stage (DOS).

ed at almost the same time as, or at an earlier stage than, contact in the anterior region in some subjects. The temporal tongue contact pattern in each region agreed with the study of Ono et al, ${ }^{10}$ in which tongue contact patterns during swallowing were investigated by placing seven pressure sensors in the experimental palatal plate. The tongue apex and the lateral part of the tongue should make contact with the anterior and lateral part of the palate, respectively, to make a concave shape in the midline of the dorsum of the tongue for the purpose of transporting a bolus to the pharynx, so the tongue may first contact the anterior region and then the anterior-lateral region of the palate. In addition, the pattern of tongue contact with the palate when swallowing may involve functional adjustment of the shapes of the tongue and palate, which leads to individual differences and may be influenced by the viscosity and volume of food. ${ }^{8}$ Further studies are required to address these is-

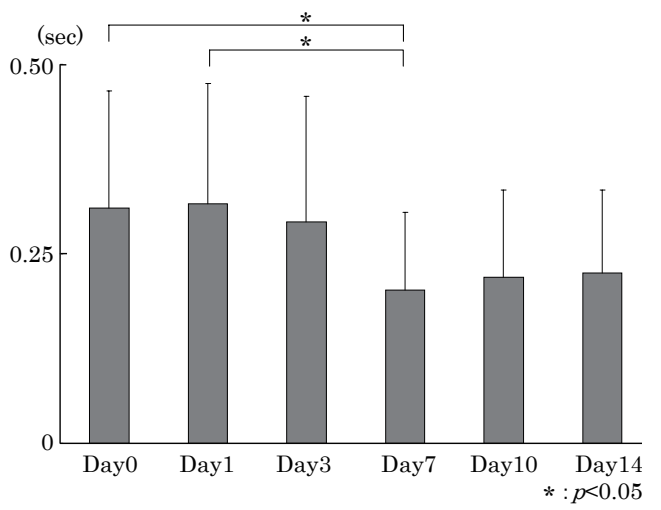

Fig. 5 Temporal change in duration of oral preparatory stage (DOPS).

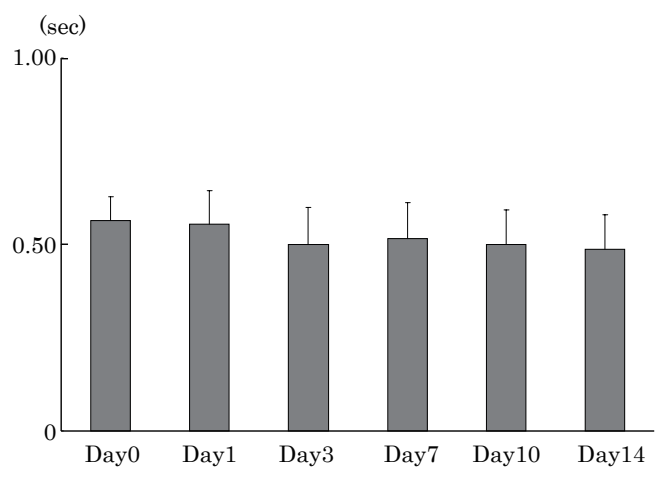

Fig. 7 Temporal change in duration of pharyngeal stage (DPS).

sues. Similar results were observed on all measurement days. We note that the experimental palatal plate used in this study was only $2-\mathrm{mm}$ thick, and this may not have changed the order of tongue palate contact during swallowing. In a future study, a more detailed investigation of this issue is required, including an examination of the effect of the thickness of the palatal plate in different regions.

\section{Temporal changes in the duration of swallow- ing}

DTS was significantly reduced on and after Day 3 (compared to Day 0) and stabilized on and after Day 7. DTS on and after Day 7 was similar to the times of $1.10 \mathrm{sec}$ reported by Shaw et $\mathrm{al}^{3}$ (observation by $\mathrm{VF}$ of swallowing of $2 \mathrm{ml}$ of a barium sulfate) and $1.08 \mathrm{sec}$ found by Hrycyshyn et $\mathrm{al}^{25}$ (observation of EMG activity of the suprahyoid muscles). A prolonged duration of swallowing may 
indicate difficulty with swallowing, and several studies suggest that the duration of swallowing in the elderly is longer than that in younger adults because of an age-related reduction in swallowing function..$^{2-4}$ Therefore, the palate covering temporarily lowered the swallowing function in this study, but this effect was reduced by habituation to the palate covering on and after Day 7 .

The DOPS indicates the time the tongue apex picks up food from the floor of the oral cavity proper to the time when the apex contacts the anterior part of the palate. In this study, DOPS showed a tendency to shorten slightly over time, suggesting that the covering has only a small effect on tongue movement in the oral preparatory stage. The presence of the upper anterior teeth as a guide, rather than the effects of the palate covering, is of importance for the tongue function in the oral preparatory stage. In elderly patients, the tongue apex did not contact the anterior part of the palate and protruded from the oral cavity proper when the subjects did not wear their upper complete dentures, which resulted in prolongation of the oral preparatory stage. ${ }^{11}$ The DOS indicates the duration from the time the tongue apex contacts the anterior part of the palate to the time when the bolus is transported to the pharynx, and this period significantly shortened over time. In contrast, the duration of the pharyngeal stage (DPS) did not show any significant changes over time. Voluntary movement occurs in the oral stage, whereas reflex movement is observed in the pharyngeal stage. Therefore, the palate covering may mainly influence the oral stage, rather than the pharyngeal stage. However, Ohmae et $\mathrm{al}^{26} \mathrm{re}^{-}$ ported that changes in the anchor function of the tongue apex may have an effect on the contact between the tongue base and the posterior pharyngeal wall, resulting in effects on the pharyngeal stage. In the current study, the palate covering has an effect on the oral stage, but may have a secondary effect on the pharyngeal stage. The palate covering interfered with sensory nerve endings in the palate and changed the volume of the oral cavity and the shape of the palate, interfering with smooth contact between the tongue and the palate and prolonging the swallowing time. However, habituation to the palate covering and learning of the new pattern of tongue contact with the palate resulted in shortening of the oral stage over time, which contributed to shortening the overall duration of swallowing.

This study was limited to young and healthy dentulous subjects, suggested that the influence of the palate covering on swallowing function is relieved over a short time (approximately 7 days). Our results supported several studies using an experimental palatal plate suggested that it needed 3-7days ${ }^{16-18}$ to habituate to the articulation with a palate covering. Correspondingly, in terms of swallowing, our results were similar to the 3 days over reported by Ando et al ${ }^{19}$ (observation by surface EMG of suprahyoid and infrahyoid muscles of swallowing following mastication). Therefore, the commonly used approach in the elderly with dysphagia, such as avoiding dentures due to their disadvantages, should be reconsidered. However, there are many factors related to habituation to cover a plate, such as age, types of edentulous jaw, food viscosity and consistency for swallowing, denture design, time for wearing, et al. In a future study, a more detailed investigation is required.

\section{Conclusion}

The duration of total swallowing was significantly shortened on and after Day 3, compared to the value immediately after placement of the experimental palatal plate covering, and stabilized on and after Day 7. This change was mainly caused by significant shortening of the oral stage of swallowing which reflected voluntary movement. In contrast, there were no significant changes over time in the duration of the pharyngeal stage of swallowing, which reflects reflex movement.

Acknowledgements: This work was supported, in part, by Grants-in-Aid for Young Scientists (Start-up) (18890179) (2006-2007), and High-Tech Research Project (2005-2009) from the Ministry of Education, Culture, Sports, Science and Technology of Japan. Part of the work was presented at the 115th Scientific Meeting of the Japan Prosthodontic Society and the 18th Meeting of the Japanese Society of Gerodontology and the 12th Meeting of the Japanese Society of Dysphagia Rehabilitation.

\section{References}

1. Yoshikawa M, Yoshida M, Nagasaki T el al. Influence of aging and denture use on liquid swallowing in healthy dentulous and edentulous older people. JAGS 2005; 54: 444-449.

2. Robbins J, Hamilton JW, Lof GL et al. Oropharyngeal swallowing in normal adults of different ages. Gastroenterology 1992; 103: 823-829.

3. Shaw DW, Cook IJ, Gabb M et al. Influence of nor- 
mal aging on oral-pharyngeal and upper esophageal sphincter function during swallowing. Am J Physiol 1995; 268: G397-G403.

4. Tracy JF, Logemann JA, Kahrilas PJ et al. Preliminary observation on the effects of age on oropharyngeal deglutition. Dysphagia 1989; 4: 9094.

5. Sheth N, Diner WC. Swallowing problems in the elderly. Dysphagia 1988; 2: 209-215.

6. Feinberg MJ. Radiographic techniques and interpretation of abnormal swallowing in adult and elderly patients. Dysphagia 1993; 8: 356-358.

7. Leopold NA, Kagel MC. Swallowing, ingestion and dysphagia: A reappraisal. Arch Phys Med Rehabil 1983; 64: 371-373.

8. Kahrilas PJ, Lin S, Logemann JA et al. Deglutitive tongue action: volume accommodation and bolus propulsion. Gastroenterology 1993; 104: 152-162.

9. Sonies BC, Parent LJ, Morrish K et al. Durational Aspects of the Oral-Pharyngeal Phase of Swallow in Normal Adults. Dysphagia 1988; 3: 1-10.

10. Ono T, Hori K, Nokubi T. Pattern of tongue pressure on hard palate during swallowing. Dysphagia 2004 ; 19 : 259-264.

11. Furuya J. Effects of wearing complete dentures on swallowing in the elderly. J Stomatol Soc 1999; 66: 361-369. (in Japanese)

12. Doods WJ, Taylor AJ, Stewart ET et al. Tipper and dipper types of oral swallows. Am J Roentgenol 1989; 153: 1197-1199.

13. Nagao K, Kitaoka N, Kawano F et al. Influence of changes in occlusal vertical dimension on tongue pressure to palate during swallowing. Prosthodont Res Pract 2002; 1: 16-23.

14. Nagaosa S, Mukai Y. A general tongue pressure measuring method which does not depend only on the maximum tongue pressure. JJDR 2005; 9:139-147. (in Japanese)

15. Ishigami $\mathrm{H}$. Phonetical investigation of habituation on experimental palatal plate wearer's utter- ances. J Jpn Prosthodont Soc 1994; 38:683-695. (in Japanese)

16. Hagiwara M. Relation between articulatory function and prosthesis. J Jpn Prosthodont Soc 1990; 34: 467-479. (in Japanese)

17. Yamada S. An experimental study on changes in tongue pressure during speech articulation. J Osaka Odont Soc 1984; 47: 68-87. (in Japanese)

18. Betto S. An experimental study on full palatal plate as to phonetic adaptation. J Osaka Odont Soc 1974; 37: 557-591. (in Japanese)

19. Ando R, Nakamura K, Masumi S. Effects of wearing palatal plate on swallowing and its habituation. J Jpn Prosthodont Soc 2007; 51: 760-767. (in Japanese)

20. Cook IJ, Dodds WJ, Dantas RO et al. Timing of videofluoroscopic, manometric events, and bolus transit during the oral and pharyngeal phases of swallowing. Dysphagia 1989; 4: 8-15.

21. Shaker R, Dodds WJ, Dantas RO et al. Coordination of deglutitive glottic closure with oropharyngeal swallowing. Gastroenterology 1990; 98: 1478-1484.

22. Hiiemae KM, Palmer JB. Food transport and bolus formation during complete feeding sequences on foods of different initial consistency. Dysphagia $1999 ; 14: 31-42$.

23. Taniguchi H, Inoue M, Yamada Y. Effects of food consistency and posture on swallowing function in man. J Jpn Soc Stomatognath Funct 2006; 12: 132-133. (in Japanese)

24. Reimers NL, Logemann J, Larson C. Viscosity effects on EMG activity in normal swallow. Dysphagia 1994; 9:101-106.

25. Hrycyshyn AW, Basmajian JV. Electromyography of the oral stage of swallowing in man. Am J Anat 1972; 133:333-340.

26. Ohmae Y, Ogura M, Karaho T et al. Effects of anchoring function on tongue tip during oropharyngeal swallow. Otologia Fukuoka 1998; 44: 301304. (in Japanese) 